\title{
Ambientes Educacionais Colaborativos com Realidade Aumentada
}

\author{
Ezequiel Roberto Zorzal ${ }^{1}$, PPGEE/UFU, CEFET-SP, ezorzal@gmail.com \\ Claudio Kirner, PPGCC/UNIMEP, ckirner@unimep.br \\ Alexandre Cardoso, PPGEE/UFU, alexandre@ufu.br \\ Edgard A. Lamounier Jr. PPGEE/UFU, lamounier@ufu.br \\ Mônica Rocha Ferreira de Oliveira, PPGEE/UFU, monica-rocha@ hotmail.com \\ Luciano Ferreira Silva, PPGEE/UFU, DCC/UFRR, luciano@mat.ufu.br
}

Resumo: A aprendizagem colaborativa presencial e remota vem sendo usada cada vez mais como uma abordagem educacional eficiente. A evolução tecnológica permite, atualmente, replicar e, até mesmo, amplificar características da comunicação interpessoal, mas o problema da manipulação remota de objetos continua sendo difícil de ser resolvido. No entanto, a Realidade Virtual e Realidade Aumentada permitem a manipulação de objetos virtuais de maneira parecida com as situações reais. Assim, este trabalho discute essas questões e apresenta soluções para a interação em ambientes colaborativos presenciais e remotos, usando recursos para a comunicação interpessoal (chat, áudio e vídeo-conferência) e Realidade Aumentada.

Palavras-chave: Realidade Aumentada, Ambientes Colaborativos, Interação.

\section{Collaborative Education Environments with Augmented Reality}

Abstract: The face-to-face and remote collaborative learning has been used more and more as an efficient educational approach. The technological evolution allows, now, to replicate and, even, to amplify characteristics of the interpersonal communication, but the remote manipulation of objects remains a problem to be solved. However, the Virtual Reality and Augmented Reality make possible the manipulation of virtual objects in a way similar to real situations. This work discusses those subjects and presents solutions for interactions on face-to-face and remote collaborative environments, using resources for the interpersonal communication (chat, audio and video-conference) and Augmented Reality.

Keywords: Augmented Reality, Collaborative Environments, Interaction.

\section{Introdução}

A aprendizagem colaborativa, como uma abordagem educacional centrada nos estudantes e baseada em trabalhos em grupo, vem ganhando espaço ao longo dos anos (Smith, 1992). Essa abordagem leva em conta que: o aprendizado é ativo, através de processo construtivo; depende de contextos mais ricos; e envolve estudantes diversificados. Assim, procura-se situações, nas quais o aluno possa envolver-se, trabalhar em grupo, competir e cooperar, além de exercitar o conceito de responsabilidade.

Pode-se implementar a aprendizagem colaborativa e o desenvolvimento de habilidades em grupo, tanto em ambientes presenciais (face-a-face), quanto em ambientes remotos suportados por computador (Billinghurst et al., 2003).

A grande vantagem da colaboração presencial está na facilidade de interação entre pessoas que utilizam comunicação verbal, gestos, expressões faciais e movimentos naturais para a manipulação de objetos. Quando a aplicação passa para o âmbito do

\footnotetext{
1 "Bolsista do CNPq - Brasil"
} 
computador no mesmo ambiente, boa parte dessas características persiste, mas a manipulação dos objetos é alterada, exigindo-se uma interface e dispositivos de interação que requer adaptação do usuário. Ao colocar os usuários para atuarem remotamente, as vantagens presenciais diminuem, mas o alcance das aplicações faz com que se procure superar essas dificuldades. Nesse caso, técnicas de comunicação multimídia, envolvendo texto, voz, vídeo e animação são usadas para replicar e potencializar as características presenciais. No entanto, os problemas da manipulação de objetos continuam sendo os mais difíceis de se resolver.

Para viabilizar a manipulação de objetos de uma maneira mais natural, usa-se a Realidade Virtual que permite implementar interfaces tridimensionais, propiciando visualização e manipulação parecidas com as ações no mundo real, exigindo, no entanto, dispositivos especiais como luvas e capacetes, dentre outros. Isto se deve ao fato do usuário ter que entrar no contexto da aplicação executada dentro do computador, exigindo que ele conheça, ou seja, treinado no uso desses dispositivos especiais (Kirner, Tori, 2004). Assim, apesar dos benefícios de uma interação mais natural, a necessidade de equipamentos especiais e de treinamento para seu uso acaba limitando o alcance da Realidade Virtual.

Uma solução para esse tipo de problema foi dada pela tecnologia de Realidade Aumentada que, misturando o cenário real com objetos virtuais gerados por computador, produz um único ambiente, sobreposto ao ambiente físico disposto na frente do usuário. Além disso, o usuário, utilizando as mãos, consegue manipular os objetos reais e virtuais do cenário misturado, sem a necessidade de equipamentos especiais. Portanto, a Realidade Aumentada, ao ser usada em conjunto com o suporte de comunicação existente hoje em dia, participa de uma convergência de recursos multimídia que permite, às pessoas remotamente localizadas, usufruírem das vantagens do trabalho presencial (face-a-face), incluindo os vários tipos de interação, mesmo estando em ambientes remotos.

Isso propicia ambientes colaborativos diversos, incluindo a exploração dos benefícios dos jogos educacionais, em função de suas características lúdicas, de envolvimento, de desenvolvimento de habilidades e de construção do conhecimento.

Desta maneira, este trabalho apresenta as características, recursos e aplicações educacionais de Realidade Aumentada e mostra a implementação de dois jogos educativos em ambiente colaborativo com Realidade Aumentada para justificar as vantagens salientadas e ressaltar seu potencial de utilização.

Assim, a Seção 2 apresenta os conceitos de Realidade Aumentada, a Seção 3 discute os ambientes colaborativos de Realidade Aumentada, enquanto a Seção 4 mostra as aplicações colaborativas desenvolvidas pelo Grupo de Realidade Virtual da Universidade Federal de Uberlândia. Finalmente, na seção 5, são apresentadas as conclusões.

\section{Realidade Aumentada}

A Realidade Aumentada é uma particularização de um conceito mais geral, denominado Realidade Misturada, que consiste na sobreposição de ambientes reais e virtuais, em tempo real, através de um dispositivo tecnológico. Uma das maneiras mais simples de se conseguir isto baseia-se no uso de um microcomputador com uma webcam, executando um software que, por meio de técnicas de Visão Computacional e processamento de imagens, mistura a cena do ambiente real, capturada pela webcam, 
com objetos virtuais gerados por computador. O software também cuida do posicionamento, oclusão e interação dos objetos virtuais, dando a impressão ao usuário que o cenário é único.

A Realidade Misturada pode receber duas denominações: Realidade Aumentada, quando o ambiente principal ou predominante é o mundo real e, Virtualidade Aumentada, quando o ambiente principal ou predominante é o mundo virtual (Azuma et al., 2001). Assim, a Realidade Aumentada pode ser definida como a sobreposição de objetos virtuais no mundo real, por meio de um dispositivo tecnológico, melhorando ou aumentando a visão do usuário (Bajura, Neumann, 1995; Azuma, 1997; Milgram et al., 2004). Cabe ressaltar que, neste caso, os objetos virtuais são trazidos para o espaço do usuário, onde ele tem segurança e sabe como interagir, sem a necessidade de treinamento. Além disso, o usuário com uso das mãos, consegue manipular os objetos reais e virtuais do ambiente misturado, sem a necessidade de equipamentos especiais.

Esta tecnologia permite que o usuário tenha uma interação atrativa e motivadora com tais ambientes e por conseqüência, o desenvolvimento de habilidades e a construção do conhecimento. Entretanto, para que esses objetos sobrepostos sejam visualizados, é necessário a utilização de algum software e dispositivos tecnológicos.

Para o desenvolvimento das aplicações deste artigo utilizou-se a ARToolKit (Augmented Reality Toolkit) (Kato et al., 2000), uma biblioteca, com código aberto e gratuita, apropriada para desenvolver aplicações de Realidade Aumentada, que faz uso de técnicas de Visão Computacional para o reconhecimento de padrões e inserção dos objetos virtuais no ambiente real.

A estratégia de concepção de soluções com uso da ARToolKit fundamenta-se em um conjunto de procedimentos. Inicialmente, a imagem capturada pela câmera é transformada em valores binários (Preto \& Branco). Essa imagem é analisada pelo software, procurando por regiões quadradas que possam indicar a existência de uma marca. Assim, quando uma marca (marcador) é reconhecida, ele examina o interior da mesma fazendo uma busca pelo símbolo desenhado. Em seguida, este símbolo é capturado e comparado com os símbolos pré-cadastrados na biblioteca. Por fim, se for encontrada alguma similaridade entre símbolos capturados com símbolos précadastrados, é considerado então que foi encontrado um dos padrões de referência. Dessa forma, a ARToolKit utiliza o tamanho conhecido do quadrado e a orientação do padrão encontrado para calcular a posição real da câmera em relação a posição real do marcador. Assim, uma matriz $3 \times 4$ é gerada, contendo as coordenadas reais da câmera em relação ao marcador. Esta matriz é usada para calcular a posição das coordenadas da câmera virtual. Se as coordenadas virtuais e reais da câmera forem iguais, o objeto virtual é desenhado precisamente sobre o marcador real.

A Realidade Aumentada, pelo fato de permitir trabalhar com objetos virtuais no espaço tridimensional, traz um grande potencial de ampliação dos limites dos jogos tradicionais, rompendo a barreira da gravidade e permitindo posicionar peças no espaço, sem a necessidade de elementos auxiliares.

\section{Ambientes Colaborativos com Realidade Aumentada}

Por muitas décadas foram desenvolvidas pesquisas voltadas para o uso do computador em atividades colaborativas, envolvendo principalmente participantes remotos. A área de trabalhos colaborativos suportados por computador (CSCW) possui inúmeros exemplos de aplicações nesse sentido (Billinghurst, Kato, 2002; Billinghurst 
et al., 2003), envolvendo áudio e vídeo-conferência, sistemas colaborativos de Realidade Virtual, etc.

No entanto, para conseguir-se a colaboração por computador, incluindo a manipulação natural de objetos, foram desenvolvidas mais recentemente interfaces com Realidade Aumentada. Essas interfaces abrangem colaboração face-a-face e colaboração remota, envolvendo objetos reais e virtuais.

A colaboração face-a-face com Realidade Aumentada (Schmalteig et al., 1996) é baseada no compartilhamento do ambiente físico, misturado com objetos virtuais e visto através de capacete ou no monitor. Os participantes do trabalho colaborativo atuam nos objetos reais e virtuais do mesmo ambiente, tendo cada um sua visão, quando usam capacete com microcâmera, ou a mesma visão, quando se usa monitor com webcam. Ambas possibilidades utilizam visão baseada em vídeo por ser mais popular.

A Figura 1 mostra exemplos de colaboração face-a-face com Realidade Aumentada com câmera de vídeo acoplada ao capacete e com monitor.

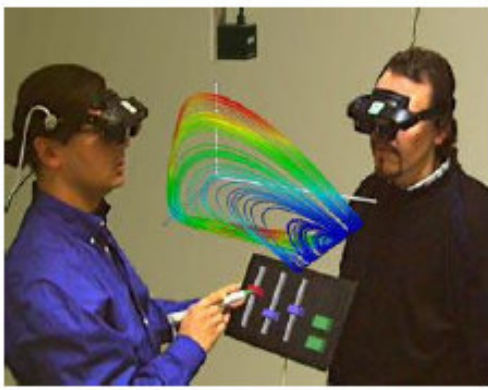

Com capacete

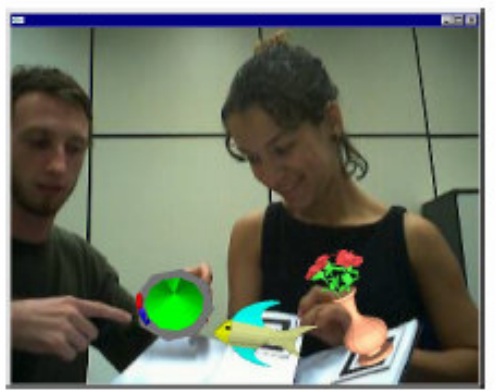

Com monitor

Figura 1. Colaboração face-a-face com visão baseada em vídeo, usando capacete ou monitor (Schmalteig et al., 1996).

No caso do uso do capacete, a interface é bastante intuitiva e voltada para a colaboração no mundo real, em função da manutenção dos protocolos sociais decorrentes da característica presencial.

As principais características desse ambiente colaborativo com Realidade Aumentada são: virtualidade, aumento, cooperação, independência e individualidade. Como os objetos virtuais possuem características parecidas com os objetos reais, como dimensão e posicionamento, eles podem ser manipulados com técnicas tangíveis de Realidade Aumentada, como toque, transporte, etc.

No caso da colaboração face-a-face com Realidade Aumentada, usando visão baseada em monitor e webcam, os usuários manipulam os objetos, visualizando-os no monitor, perdendo assim as características de independência e individualidade. A interface é muito parecida com a anterior, exceto que todos os usuários possuem o mesmo ponto de vista, mostrado no monitor, e que a visualização, em vez de ocorrer no espaço físico, onde estão as mãos, ocorre no monitor.

A colaboração remota com Realidade Aumentada, por sua vez, baseia-se em interfaces computacionais que compartilham informações e sobrepõem os espaços físicos dos vários usuários (mesa, por exemplo), utilizando uma rede de computadores. Assim, cada usuário pode colocar objetos virtuais sobre a mesa, de forma a visualizar todo o conjunto de objetos e manipulá-los. Usando ARToolKit, cada usuário poderá 
colocar suas placas no campo de visão da webcam, enxergando seus objetos e os objetos dos outros que aparecem no cenário, em função de suas posições, promovendo assim a colaboração remota.

O uso integrado de ferramentas de áudio e vídeo-conferência com interação em ambientes de Realidade Aumentada permite a implementação de ambientes colaborativos remotos com as vantagens da colaboração face-a-face.

O sucesso da aplicação depende de potência de processamento para dar conta da execução do software de Realidade Aumentada e da velocidade da rede para permitir a comunicação e interação em tempo real. Os microcomputadores atuais possuem potência computacional adequada para as aplicações de Realidade Aumentada. O gargalo está na rede para sustentar os requisitos de fluxo dos elementos multimídia (áudio e vídeo). A solução envolve a melhoria das instalações e/ou de procedimentos que minimizem o tráfego e superem problemas de atraso. Atualmente, em algumas situações, onde a banda larga já é realidade, essas aplicações funcionam bem, mas para a sua universalização, ainda há muito que fazer. Enquanto isso, soluções múltiplas, que permitam a convivência com a heterogeneidade de computadores e de comunicações, estão sendo desenvolvidas para oferecerem qualidades diferentes compatíveis com os recursos disponíveis em cada caso.

\section{Ambientes Desenvolvidos}

Esta seção apresenta dois jogos colaborativos, o primeiro requer uma colaboração remota e o segundo faz-se uso de colaboração face-a-face.

\subsection{Jogo da Velha Colaborativo}

Desenvolveu-se um jogo da velha em ambiente de Realidade Aumentada utilizando dois computadores remotos.

$\mathrm{Na}$ implementação, a comunicação em rede baseou-se em sockets. Paralelamente, a ARToolKit foi utilizada para gerar todo ambiente do jogo e permitir o compartilhamento do ambiente físico dos usuários, de forma que todos pudessem trabalhar no mesmo espaço.

O funcionamento do jogo da velha virtual é similar ao jogo convencional. Um dos participantes cria o tabuleiro virtual, que fica disponibilizado para o outro participante remoto, e o jogo se inicia. Os participantes seguem colocando suas peças até que um deles forme uma seqüência de três símbolos adjacentes na horizontal, vertical ou diagonal.

Quando isso acontece, o participante faz uso de uma régua virtual indicando sua vitória, conforme a Figura 2.
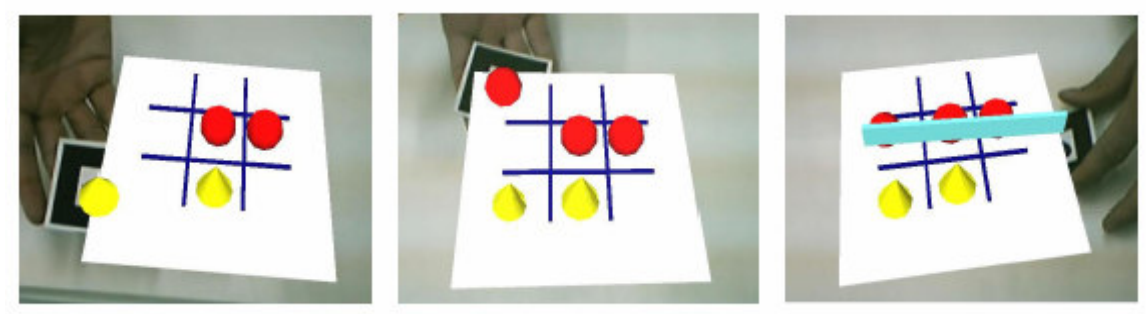

Figura 2. Etapas do jogo da velha com Realidade Aumentada em rede. 
Este jogo proporciona uma interação não apenas virtual, mas também humana, visto que os participantes podem jogar com oponentes humanos, sem a interferência do computador. Existe, ainda, a possibilidade de o computador participar do processo, ajudando a identificar ganhadores e estabelecendo quem joga.

Assim, este tipo de jogo permite que seu usuário estabeleça oportunidades de encontrar soluções e interagir com outros usuários, possibilitando então o processo de atividades colaborativas e ampliando estratégias coletivas.

A Figura 3 apresenta a estrutura e ambiente do jogo da velha colaborativo.

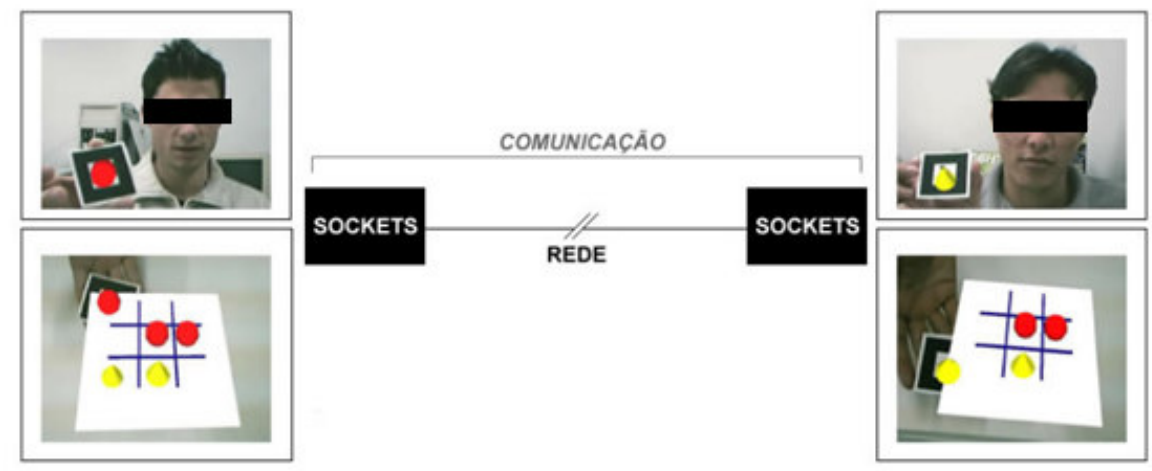

Figura 3. Estrutura do jogo da velha colaborativo.

Paralelamente a essas ações, os usuários poderão trocar mensagens de texto na janela de chat, conversar por meio do canal de voz e ver-se através de uma janela de vídeo. Dependendo da qualidade da rede e da situação de tráfego, alguns desses elementos de apoio poderão ser desativados para que o sistema funcione a contento. $\mathrm{O}$ único elemento que não pode ser desativado é a aplicação de Realidade Aumentada, que constitui-se no jogo em si e que, potencialmente, exige menos tráfego na rede.

Para isto, foram feitos alguns ajustes no sistema, visando minimizar o tráfego de informações do jogo. Inicialmente, as informações de posições do tabuleiro e das peças eram enviadas continuamente para garantir a consistência das posições para os usuários remotos. Isto provocava um tráfego intenso e desnecessário de informações na rede.

Para diminuir este tráfego, foi colocado um programa analisador de posições que armazena a posição anterior e atual das posições coletadas pelo software de Realidade Aumentada. Se as posições (X, Y ou Z) do tabuleiro ou das peças não diferirem de uma tolerância, por exemplo, de $0,5 \mathrm{~cm}$, as informações não precisarão ser enviadas. A cada leitura, faz-se a análise e transforma-se a posição atual em anterior para a análise seguinte. Assim, as informações só são enviadas, quando efetivamente há alterações de posicionamento do tabuleiro ou das peças. Assim, com um tráfego pequeno de informações curtas, a rede, de maneira geral, suporta a aplicação de Realidade Aumentada, mesmo em condições de baixa velocidade.

\subsection{Batata-Quente}

No jogo original, algumas pessoas se reúnem em forma de roda, enquanto uma delas, de costas para as demais, repete a frase: "Batata Quente", por algum tempo e depois diz: "Queimou!". 
Enquanto a primeira frase é repetida, as pessoas passam entre si um objeto qualquer, representando a batata. Quem estiver com o objeto no momento que foi dito: "Queimou!" é eliminado do jogo. Este processo se repete até restar apenas um jogador.

Esta ação exercita as habilidades motoras das pessoas, ao mesmo tempo em que acrescenta um certo nível de euforia, pois nenhum dos participantes deseja ser eliminado da partida.

Apesar de todas essas vantagens, esse jogo apresenta algumas deficiências que o impedem de ser mais atrativo. Um deles é o fato de o objeto ser estático, não refletindo a proposta titulada, que seria a batata queimar.

A presente aplicação buscou formas de enriquecer o jogo original, visando melhorar o nível de excitação imposto aos jogadores através de técnicas de Realidade Aumentada.

Um objeto virtual representa a batata, mudando sua forma e cor quando o estado de queimado ocorre. A forma de movimentação do objeto também foi enriquecida e inovada, utilizando Realidade Aumentada.

Para esse jogo foi utilizado um carrinho de controle remoto, por intermédio de uma câmera sem fio instalada nele, obtém as imagens da pista e transmite para o computador. Para cada imagem, o computador faz uma análise a fim de se detectar os marcadores de controle (placas de sinalização). Os marcadores são utilizados para a identificação dos comandos a serem executados. Os seguintes comandos podem ser utilizados pelos jogadores: seguir em frente, virar a esquerda, virar a direita. Quando um marcador é encontrado na imagem, o comando associado a ele é enviado para o carrinho, conforme Figura 4.

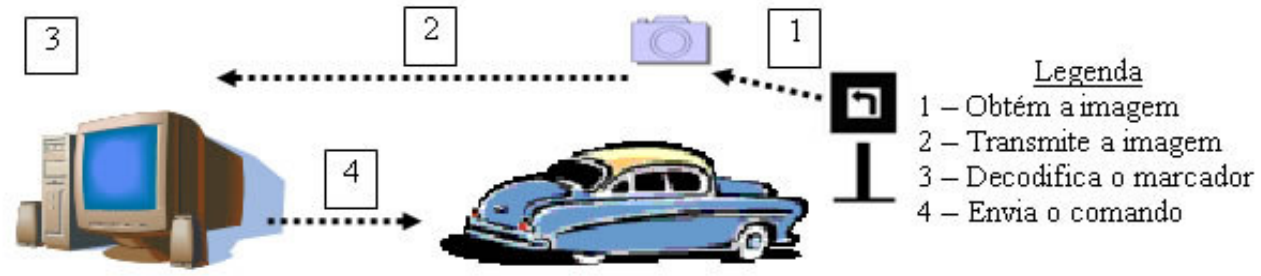

Figura 4. Funcionamento do carrinho.

Para isto, o dispositivo de controle remoto do veículo foi ligado à porta paralela de um computador, o que exigiu o desenvolvimento de um circuito. Este circuito recebe os sinais elétricos (comandos de controle do carrinho) da porta paralela e os envia para o controle remoto, o qual transmite para o carrinho.

Esse carrinho deverá ser controlado pelos participantes numa espécie de tabuleiro através de marcadores pré-cadastrados que permitem que os determinados comandos sejam executados. A Figura 5 apresenta os marcadores cadastrados e seus respectivos comandos. 


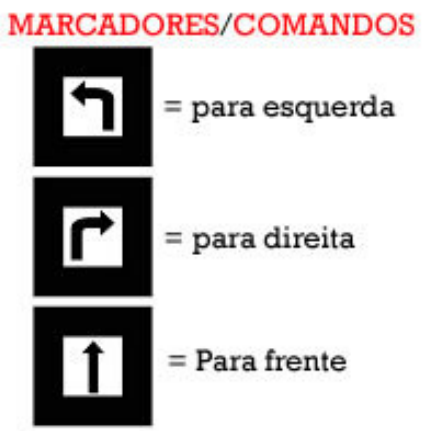

Figura 5. Marcadores cadastrados.

Sobre o carrinho é colocado um marcador de referência (Figura 6 (a)), que ao ser posto no campo de visão da câmera apresentará uma batata virtual sobreposta a ele. A batata virtual poderá ser visualizada no monitor ou no capacete do usuário.

O objetivo dos participantes é conduzir o carrinho contendo a batata virtual para o campo do adversário, conforme a Figura 6 (b). O tempo de duração pode ser aleatório aumentando a sensação de incerteza nos jogadores e motivando-os a se livrarem do carrinho, conduzindo-o o mais rápido possível para o campo do adversário.

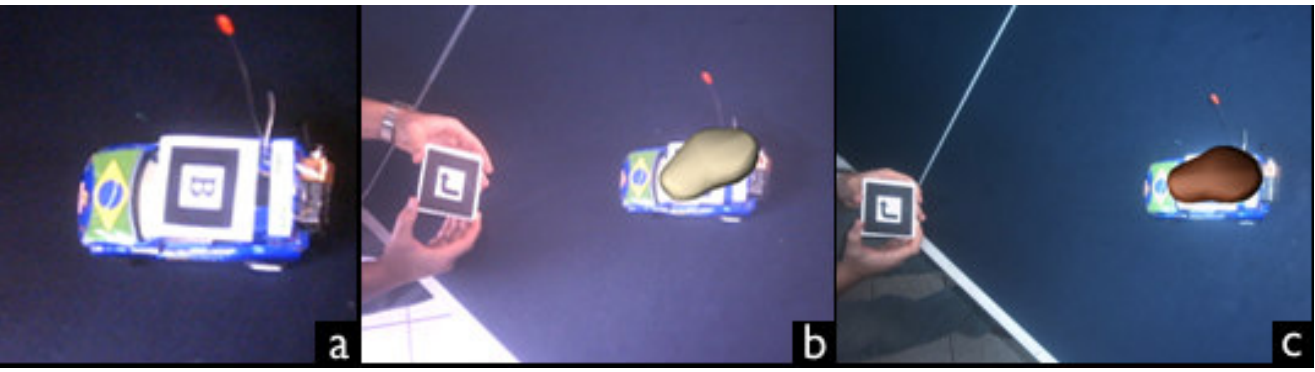

Figura 6. Ambiente do jogo.

No momento em que a batata queima, o carrinho pára de se movimentar e o objeto virtual em cima dele muda de aparência, conforme Figura 6 (c). Assim, é indicado o vencedor da partida de uma forma interativa e atrativa.

\section{Conclusões}

O emprego de Realidade Aumentada em ambientes educacionais contribui de maneira significativa na percepção, interação e motivação dos usuários. Ela garante um grande potencial na criação de ambientes colaborativos, permitindo uma interação natural de fácil adaptação e livre de dispositivos especiais.

Este artigo discutiu ambientes colaborativos com Realidade Aumentada e mostrou que é possível utilizar esta tecnologia para criar ambientes colaborativos remotos e presenciais, gerando dessa forma ambientes agradáveis, motivadores, planejados e enriquecidos, para estimular a aprendizagem e o desenvolvimento de habilidades em grupo.

Os jogos em grupo são importantes e propiciam aos envolvidos diversos benefícios, como por exemplo: noções de cooperação, aprendizado de como agir e se portar numa sociedade, limites de liberdade, entre outros. Esses tipos de jogos possuem diversas vantagens, já que é permitida uma maior facilidade de interação entre pessoas 
que utilizam comunicação verbal, gestos, expressões faciais e movimentos naturais para manipulação de objetos.

Os jogos com Realidade Aumentada têm um potencial muito grande de desenvolvimento, que até agora foi pouco explorado. A área de trabalhos colaborativos, especificamente, apresenta um espaço interessante de desenvolvimento, em função da existência de múltiplos usuários interagindo em um mesmo espaço compartilhado. Esses ambientes exigirão novas formas de interação, gerando novas interfaces que deverão facilitar o trabalho das pessoas e o seu desenvolvimento cognitivo. O Grupo de Realidade Virtual da Universidade Federal de Uberlândia tem trabalhado no desenvolvimento de diversos ambientes educacionais utilizando Realidade Aumentada, e atualmente trabalha em testes dos mesmos.

\section{Agradecimentos}

Os Autores agradecem ao Conselho Nacional de Desenvolvimento Científico e Tecnológico (CNPq - Brasil) e à Coordenação de Aperfeiçoamento de Pessoal de Ensino Superior (CAPES) pelo apoio financeiro oferecido através do Programa de Doutorado no País.

\section{Referências}

AZUMA, R. T. (1997) "A Survey of Augmented Reality", Presence: Teleoperators and Virtual Environments, v.6, n.4, p. 355-385.

AZUMA, R. T. et al. (2001) "Recent Advances in Augmented Reality", IEEE Computer Graphics and Applications, v .21, n.6, p. 34-47.

BAJURA, M.; NEUMANN, U. (1995) "Dynamic Registration Correction in VideoBased Augmented Reality Systems" IEEE Computer Graphics \& Applications, v.15, n.5. p.52-60.

BILLINGHURST, M., BELCHER, D., GUPTA, A., \& KIYOKAWA, K. (2003) "Communication behaviors in co-located collaborative AR interfaces", International Journal of Human-Computer Interaction, v. 16, n.3, p. 395-423.

BILLINGHURST, M.; KATO, H. (2002) "Collaborative augmented reality", Communications of the ACM, v.45, n.7, p. 40-44.

KATO, H.; BILLINGHURST, M.; POUPYREV, I. ARToolKit version 2.33 Manual, Novembro. 2000.

KIRNER, C.; TORI, R. (2004) "Introdução à Realidade Virtual, Realidade Misturada e Hiper-realidade", In: Claudio Kirner; Romero Tori. (Ed.). Realidade Virtual: Conceitos, Tecnologia e Tendências. 1ed. São Paulo, v. 1, p. 3-20.

MILGRAM, P. et al. Augmented Reality: A Class of Displays on the RealityVirtuality Continuum. Telemanipulator and Telepresence Technologies, SPIE, V.2351, 1994.

SCHMALSTEIG, D., FUHRMANN, A., SZALAVARI, Z., GERVAUTZ, M., (1996) "Studierstube - An Environment for Collaboration in Augmented Reality", CVE '96 Workshop.

SMITH, B. L.; MACGREGOR, J. T. (1992). "What is collaborative learning?" In 
r

Goodsell, A. S., Maher, M. R., and Tinto, V. (Eds.), Collaborative Learning: A Sourcebook for Higher Education. National Center on Postsecondary Teaching, Learning, \& Assessment, Syracuse University. 